\title{
An experimental investigation of asymmetric contests*
}

\author{
Miguel A. Fonseca ${ }^{\dagger}$
}

October 16, 2007

\begin{abstract}
Contest theory has been used in Industrial Organization to describe phenomena like R\&D races, or efforts to defend a monopoly position. When pricing behavior is constrained by regulators, competition can also take the form of a contest. This paper reports on an experimental test of the effects of asymmetry in the Tullock contest success function. Both the simultaneousmove and sequential-move frameworks are considered. Despite high levels of overbidding across the different conditions, the introduction of asymmetries in the contest function generates experimental behavior on aggregate qualitatively consistent with the theoretical predictions. At the individual level, behavior seems divided into those subjects who bid very high amounts and those who bid very low amounts.
\end{abstract}

JEL - classification numbers: C72, C92, D72

Keywords: Laboratory experiments, rent-seeking contests, simultaneous-moves, sequentialmoves, asymmetries.

*I am grateful to Hans-Theo Normann and Anthony Heyes, a co-editor and an anonymous referee for helpful comments on earlier versions of this manuscript. All remaining errors are my own.

${ }^{\dagger}$ School of Business and Economics, University of Exeter, Streatham Court, Rennes Drive, Exeter, EX4 4PU, UK; Tel: +44 (0)1392 262584; Fax: +44 (0)1392 263242; Email: m.a.fonseca@exeter.ac.uk. 


\section{Introduction}

The problem of a set of agents spending effort in order to increase the likelihood of obtaining a prize was first put forth by Gordon Tullock (1980). In his rent-seeking model, two agents can spend part of their endowments in a lottery for a fixed prize. For a given level of effort spent by the opponent, the more effort spent in the contest means a higher likelihood of victory. However, regardless of the identity of the victor, neither player can recover her investment.

The great appeal of this model lies in its conceptual simplicity, which explains why it has been used to model a wide variety of economic problems. In markets where regulation stifles price competition, firms must procure a share of the market by lobbying regulators. Also, in industries with large switching costs like electronics, long-run survival often depends on the establishment of an industry standard. Straightforward examples are the BetaMax vs. VHS in the 1980's or BluRay vs. HD-DVD nowadays, where the hardware suppliers compete with each other in order to obtain licensing agreements with mainstream electronic firms. Another natural example of contests in I.O. is R\&D competition (Loury, 1979; Dasgupta and Stiglitz, 1980). Each dollar spent increases the likelihood of obtaining the breakthrough that leads to a patent.

Despite its simplicity, it is difficult to conduct an empirical investigation of the Tullock contest model using real world data. Data on time and resources spent in rent-seeking activities are difficult to collect. Also, the individual parameters such as relative player ability or the individual value of the prize are not usually available to researchers. For instance, in an $R \& D$ race the prize would be the discounted stream of profits over the patent's lifetime, which is a function of the firm's cost structure. This makes an empirical estimation using field data very difficult.

Experimental methods are a valuable avenue of research into the testing the predictive power of Tullock contests. The lab enables the experimenter to isolate all non-relevant factors of the decision-making process. This way, one is able to apply precise incentive structures allowing for the controlled study of the strategic aspects of the theory under scrutiny.

The experimental literature testing this type of model ${ }^{1}$ has focused mainly on symmetric, simultaneous-move contests. The main concern of this literature is to measure the extent to which subjects' actions deviate from the risk-neutral Nash equilibrium (RNNE) and the corresponding impact on rent dissipation.

\footnotetext{
${ }^{1}$ Other experimental studies of contests using functional forms other than the Tullock contest function include Coughlan and Plott (2003) who study legal battles under different fee-shifting paradigms or Schotter and Weigelt (1987) and Bull, Schotter and Weigelt (1992) who study job promotion tournaments.
} 
Millner and Pratt (1989) conducted the first experimental study of Tullock contests. In their experiment, subjects were endowed with a cash amount in every period. In each period, which lasted 90 seconds, subjects were able to purchase lottery tickets using their endowment. At the same time, they were given real-time information regarding how much of their endowment remained, as well as the number of lottery tickets purchased by the other player. Their data showed that the individual bids and rent dissipation were significantly above the RNNE. Shogren and Baik (1991) considered a single choice, private information design, where experimental subjects made their choices based on a payoff table containing the expected value of the contest, based on the bids of both players. With the new experimental design, they found that the observed rent seeking behavior was in accordance with the RNNE.

Millner and Pratt (1991) investigated the role of risk attitudes on expenditures in Tullock contests. The authors show that no general prediction can be made regarding the level of expenditure by risk-averse players without making specific assumptions about the shape of the utility function of both players. To circumvent this problem, they created an experiment in which subjects were pre-sorted with respect to their risk preferences. Subsequently, subjects would be randomly paired with people with similar risk preferences. They show that the less risk-averse groups had higher average effort and higher rent dissipation rates than more risk-averse groups. Davis and Reilly (1998) study the impact of asymmetries in the valuation of the prize in standard Tullock contests and first-price, all-pay auctions. They find that subjects bids are above the RNNE in both types of contests. However, introducing a player with a higher valuation reduced the level of overbidding.

Weimann et al. (2000) is the only paper to our knowledge to test the symmetric version of a sequential-move Tullock contest. There, each bid is raised to the power of 8 . In this parameter setup, the subgame-perfect equilibrium is where the first-mover commits to an effort level such that second-mover chooses not to participate in the contest ${ }^{2}$. Their main findings are that firstmovers bid either very aggressively or very tamely. Second-movers either play the subgame-perfect equilibrium and bid zero or they bid very high amounts that yield both players negative expected payoffs. When facing a very small bid, they either exploit the low bid or they match the first-movers' bid.

This study seeks to understand the impact of asymmetries on behavior in Tullock contests. We consider two different protocols: one where both players move simultaneously and another where

\footnotetext{
${ }^{2}$ In this paper, subjects also base their choices on a payoff table displaying expected payoffs.
} 
the timing of moves is sequential. While the focus of the literature has been on simultaneous-move games, one can argue that some contests are better modeled as sequential-move games. In an R\&D race context, it is reasonable to assume that if there is an industry incumbent, such a firm would enjoy a first-mover advantage in the development of a new product line. Also, when renewing a consulting contract, a firm may wish to deal with the company with whom it is currently working before listening to offers from other firms.

The role of asymmetries in ability is also important. While the majority of the theoretical work in the area has focused on the symmetric case (Dixit, 1987 and Leininger, 1993 are notable exceptions), one is unlikely to encounter it in the field. This may be due to differences in experience in lobbying or differing research technologies; there are many factors which lead to varying levels of ability between players in any given contest. By understanding behavior in both timing protocols, as well as the effect of asymmetry in ability, one is also able to verify whether agents are able to take advantage of the fact that they move first. Dixit (1987) examines this problem theoretically. He shows that given the opportunity to move first, the more able player does have an incentive to submit a higher effort level than in the simultaneous-move case.

Furthermore, it is very important to understand what the conditions in which a first mover can deter other players from entering the contest by committing to a sufficiently high level of effort. This is particularly relevant in the context of R\&D races, as discussed by Dasgupta and Stiglitz (1980). From a "contest designer" point of view, by understanding the interaction between player ability and the timing structure of the contest, one may be able to design a contest which maximizes player effort, or one which minimizes aggregate rent dissipation, depending on whether one is an employer organizing a job promotion tournament or if one is a government seeking to allocate grant funds. The former may be interested in maximizing aggregate effort, whilst the latter may be interested in giving the prize to the best candidate. ${ }^{3}$

This paper makes a contribution to the literature by studying within a single framework the impact of asymmetries in ability and of different timing protocols in Tullock contests. The main findings are that in both timing protocols, subjects bid levels are inconsistent with the risk-neutral Nash equilibrium, even with the introduction of asymmetries. Individuals either bid high amounts or almost drop out of the contest altogether. Aggregate effort is always above the prediction.

\footnotetext{
${ }^{3}$ One can also think of the sequential-move structure as a form of asymmetry. Based on O'Keefe et al. (1984)'s terminology, asymmetries in the ability parameter make the contest uneven, while protocols which favor one of two symmetric players, are termed unfair.
} 
Theory is nevertheless able to qualitatively predict the impact of the introduction of asymmetries on expected profitability. This leads to the conclusion that there may be a strategic incentive for a more apt agent to move before his less apt counterpart.

\section{The Model}

Following Leininger $(1993)^{4}$, we consider a two-player game, where each player selects her level of effort, $E_{i}$ in order to obtain a prize $\left(J_{i}\right)$. The value of the contest, $V_{i}$, is given by the following equation:

$$
V_{i}=p_{i} J_{i}-E_{i}, i=1,2
$$

where $p_{i}$ is the probability of victory in the contest for player $i^{5}$ and is defined as

$$
\begin{aligned}
& p_{1}=\frac{a E_{1}}{a E_{1}+E_{2}} \\
& p_{2}=\frac{E_{2}}{a E_{1}+E_{2}} .
\end{aligned}
$$

The $a$ parameter captures the relative "ability" of Player 1 vis-à-vis Player 2. If $a$ equals 1 , the game is symmetric; both players are equally able, which means that each unit of effort spent by either player results in the same increase in probability of victory. If $a$ is larger than one, Player 1 is more able than Player 2. Therefore, in order to have an equal probability of victory in the contest, Player 2 must spend $a$ times the effort incurred by Player 1 .

The timing of the decisions and the $a$ parameter are the treatment variables. In one variant of the experiment, both players make their decisions simultaneously. In the other variant, one of the players (Player 1) makes his choice in the first period, and the other (Player 2) makes her decision in the second period.

\subsection{Simultaneous Play}

The simultaneous-move game is the standard Tullock model considered in the literature; its Nash equilibrium is found when either player is selecting a strategy which is a best reply to the other

\footnotetext{
${ }^{4}$ There is a substantial body of theoretical literature which deals with this class of functions. We refer the interested reader to Lockard and Tullock (2001), who review some of the most important theoretical literature on Tullock contests.

${ }^{5}$ If $E_{1}=E_{2}=0$. then we assume $p_{1}=p_{2}=0$. This was also the case in the experiment, as per the instructions in the Appendix.
} 
player's strategy. This implies that when both parties maximize their expected value of the contest, we have a pair of reaction functions:

$$
\begin{gathered}
E_{1}=\frac{\left(J a E_{2}\right)^{\frac{1}{2}}-E_{2}}{a} \\
E_{2}=\left(J a E_{1}\right)^{\frac{1}{2}}-a E_{1}
\end{gathered}
$$

Solving them simultaneously, gives us the unique Nash equilibrium where

$$
E_{1}=E_{2}=E=\frac{a J}{(a+1)^{2}}, \forall a \neq 0
$$

Note that both players submit the same effort in equilibrium for all values of $a$. To see the intuition behind this, let us consider the simple symmetric case, where $a$ is equal to 1 . There, both players have the same marginal "benefit" of bidding, in that the same bid by both players implies the same probability of victory. As the $a$ parameter increases, the only thing that changes is the marginal probability of winning, since costs remain unaltered. Therefore, the benefited player obtains the same probability of victory by bidding a smaller amount, whilst the reverse applies for Player 2 . This means that Player 2 will therefore bid a lower amount in order to maximize the expected value of the contest, which is the always the same for both players. This also means the Nash equilibrium bid function has its highest level of $E$ when $a=1$. Also, given that in equilibrium both players' bids are equal, total rent dissipation will vary in a similar way to changes in $a$. This result generalizes to other types of contests where the contest success function is concave such as the model considered by Schotter and Weigelt (1992). It is also generally true that aggregate effort will be maximized when players are symmetric ${ }^{6}$

\subsection{Sequential Play}

When calculating the equilibria for the sequential-move game, we will employ the Subgame-Perfect Nash Equilibrium (SPNE) refinement and work by backward induction. Since the second-mover takes the first-mover's actions as given, the first-mover is therefore able to anticipate the behavior of the second-mover and selects an effort level along the reaction function of the second-mover such that it maximizes her expected payoff. Thus we have the second-mover (Player 2), maximize his expected value of the contest, given the effort level submitted by the first-mover (Player 1), ${ }^{7}$ yielding the following reaction function:

\footnotetext{
${ }^{6}$ For a detailed analysis of a general class of Contest Success Functions of which the present model is a special case, see Dixit (1987).

${ }^{7}$ We will use the terms first-mover and Player 1 interchangeably; the same applies for second-mover and Player 2.
} 


$$
E_{2}=\max \left\{\left(a J E_{1}\right)^{\frac{1}{2}}-a E_{1}, 0\right\}
$$

Player 1 realizes this and incorporates Player 2's reaction function into his expected value function:

$$
V_{1}=\frac{a E_{1}}{a E_{1}+\left(a J E_{1}\right)^{\frac{1}{2}}-a E_{1}} J-E_{1}=\left(J a E_{1}\right)^{\frac{1}{2}}-E_{1}
$$

The effort level for Player 1 in the subgame-perfect equilibrium is therefore

$$
E_{1}=\frac{a J}{4}
$$

Substituting this expression back into Player 2's reaction function gives:

$$
E_{2}=\max \left\{\frac{a J}{2}-\frac{a^{2} J}{4}, 0\right\}
$$

It is important to investigate the conditions in which the second-mover will participate in the contest. Setting the right-hand side of equation 6 equal to zero and solving for $a$ will determine at which levels of relative ability the second-mover will find it profitable to enter the contest. It is straightforward to show that $E_{2}>0$ if and only if $0<a<2$.

Once $a=2$, when each unit of effort spent by the first-mover has the same weight as two units of effort of the second-mover, the latter should not spend any effort (provided of course that $E_{1}=a J / 4$.) But how should be the first-mover's behavior be like when $a \geq 2$ ? One would be tempted to say that the first-mover should submit the same effort level as in the case of $a=2$ thereafter. This is not the case, however.

In the SPNE, Player 1 should anticipate that Player 2 will not submit any effort once $a=2$ and $E_{1}=a J / 4$. However, as $a$ increases beyond 2 a smaller amount should be required to deter Player 2 from entering since the effectiveness of the effort relative to the first-mover is decreasing accordingly. Thus we can calculate the effort level that deters Player 2 from entering the contest for values of $a$ great than or equal to 2 : $\left(a J E_{1}\right)^{\frac{1}{2}}-a E_{1}=0 \Rightarrow E_{1}=\frac{J}{a}$.

We can now define the Subgame-Perfect Equilibria as a function of $a$ :

$$
E_{1}=\left\{\begin{array}{l}
\frac{a J}{4} \text { if } a \in(0,2] \\
\frac{J}{a} \text { if } a \in(2, \infty)
\end{array}\right.
$$

and

$$
E_{2}=\left\{\begin{array}{l}
\frac{a J}{2}-\frac{a^{2} J}{4} \text { if } a \in(0,2] \\
0 \text { if } a \in(2, \infty)
\end{array}\right.
$$




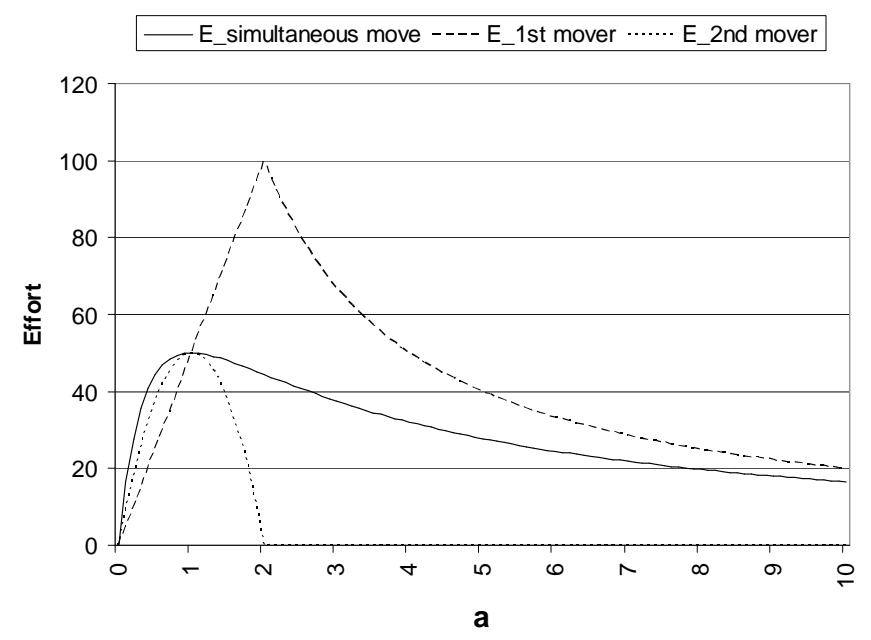

Figure 1: Equilibrium effort level in the simultaneous- and sequential-move games.

For the symmetric case, where $a=1$, both players submit the same effort level in equilibrium, i.e. $E_{1}=E_{2}=J / 4$. Hence, there is no advantage from moving first, as the probability of victory is $1 / 2$ for both players. In contrast to the simultaneous-move game, as a becomes larger than one, the behavior by either player will be markedly different. While the effort level of the second-mover immediately starts to decline monotonically, first-mover effort continues to increase until the critical point of $a=2$ is reached such that the other player does not enter the contest. At this point, the leader deters the follower from entering the contest, and as the value of $a$ approaches infinity, the equilibrium level of $E_{1}$ also approaches zero.

Figure 1 depicts equilibrium levels of effort as a function of $a$. The solid line shows the equilibrium behavior in the simultaneous-move game. The dashed line represents the first-mover's equilibrium behavior and the dotted line represents behavior by the second-mover in the sequentialmove game.

Incidentally, rent dissipation rises monotonically until $a \simeq 1.5$, when aggregate effort reaches a maximum. This is due to the fact that the rate of increase in the effort level of the leader is faster than the rate of decrease of the follower's effort level, until the critical level of $a$ is reached. Thereon, aggregate effort declines until reaching zero when $a$ approaches infinity. This implies that the contests which are fought the hardest are not the symmetric ones. Rather, the cases where aggregate effort is highest are when the first-mover has an edge over the second-mover. This prediction is also true in a more general class of contest functions, as shown by Dixit (1987). 
Before proceeding, it is worth briefly discussing the impact of departures from the standard model on equilibrium behavior. We begin with the role of risk aversion. The literature on auctions has recognized the role of risk aversion, both theoretically and experimentally (see Kagel (1995) for a review). However, the effect of risk aversion in Tullock contests is impossible to determine in the general case (Millner and Pratt, 1991; Konrad and Schlesinger 1997). Under the assumption of constant absolute risk aversion, Skaperdas and Gan (1995) show that if two players have different degrees of risk aversion, an equilibrium exists such that the player with the smallest degree of risk aversion will outbid the player with the highest degree of risk aversion. However, without making an explicit functional form for both players, we cannot generate a specific point prediction.

The second departure from the standard model is to introduce in the utility functions a concern for other players payoff. While the nature of this game may not conducive to cooperative behavior, the same is not true of competitive considerations. In other words, subjects may dislike having a lower expected payoff than their rivals. Konrad (2004) considers the case where altruistic and envious individuals compete in a perfectly discriminating contest, where the subject making the highest bid wins with certainty. He shows that equilibrium exists where altruistic and envious players earn higher payoffs than if players have standard self-interested utility functions.

We incorporate other-regarding preferences using Fehr and Schmidt (1999) model of behavior. It proposes that experimental subjects care about the differences in payoffs between themselves and the other participants in experiments and build those concerns into the standard utility function. In the current 2-player case, it would take the following form: $U_{i}=$ $V_{i}-\alpha_{i} \max \left\{V_{j}-V_{i}, 0\right\}-\beta_{i} \max \left\{V_{i}-V_{j}, 0\right\}, i \neq j$. The $\alpha_{i}$ parameter denotes aversion to disadvantageous inequality and captures the disutility a subject has from earning a smaller payoff than the other player. The authors qualify this as the players' degree of competitiveness. The $\beta_{i}$ parameter denotes aversion to advantageous inequality. A higher $\beta_{i}$ implies a more cooperative subject. Equations 9, 10 and 11 denote the equilibrium level of effort in the simultaneous-move and sequential-move cases. ${ }^{8}$ The derivation of the equilibria is the same as the standard case. Note that in all cases, if $\alpha=\beta=0$, the equilibrium effort levels are equal to the standard cases.

$$
E_{1}=E_{2}=\frac{a J(1+2 \alpha-2 \beta)}{(a+1)^{2}(1+\alpha-\beta)}, \forall a \neq 0
$$

\footnotetext{
${ }^{8}$ For simplicity, we assume $\alpha_{1}=\alpha_{2}=\alpha$ and $\beta_{1}=\beta_{2}=\beta$.
} 


$$
\begin{gathered}
E_{1}=\left\{\begin{array}{l}
\frac{a J(1+\alpha(A-2)-\beta(A+2))^{2}}{4 A((1+a)(\beta-\alpha)-1)^{2}} \text { if } a \in(0, B] \\
\frac{A J}{a} \text { if } a \in(B, \infty)
\end{array}\right. \\
E_{2}=\left\{\begin{array}{l}
\frac{a J(1+\alpha(A-2)-\beta(A+2)) \sqrt{A}}{2 A((1+a)(\beta-\alpha)-1)}-\frac{a^{2} J(1+\alpha(A-2)-\beta(A+2))^{2}}{4 A((1+a)(\beta-\alpha)-1)^{2}} \text { if } a \in(0, B] \\
\frac{A J}{a} \text { if } a \in(B, \infty)
\end{array}\right.
\end{gathered}
$$

where $A=\sqrt{\frac{1+2 \alpha-2 \beta}{1+\alpha-\beta}}$ and $B=\frac{2 \sqrt{A}(\beta-\alpha-1)}{2 \sqrt{A}(\beta-\alpha)-(1+\alpha(A-2)-\beta(A+2))}$.

Although the Fehr and Schmidt model attempts to explain behavior in a variety of games, one should take some care in applying it in the current context. Blanco et al. (2007) conduct a series of experiments in order to determine the $\alpha$ and $\beta$ parameters of the Fehr and Schmidt model. They find that although the model explains the aggregate level data well, the individual estimates fail to predict behavior across games at the individual level. The current experimental design does not allow us to estimate the parameters. Nevertheless, we will rely on existing estimates of $\alpha$ and $\beta$ to predict behavior in the game.

\section{Experimental Design and Procedures}

The experiments are designed to investigate the impact of asymmetries in two different environments: simultaneous-move and sequential-move contests. The baseline treatment will consist of symmetric treatments. This contextualizes the data with the existing literature.

The nature of the behavior required by the subgame-perfect equilibrium refinement in the sequential-move game provides a clear cut test of the theory by predicting the exclusion of the second-mover from the contest if the first-mover is sufficiently more able. We test this by setting $J_{i}=200$ and $a=7 / 3 ;^{9}$ the choice of values for $J_{i}$ and $a$ was set in order that there is a clear difference in predicted efforts in both treatments. Furthermore, the choice of $a$ was intended such that the equilibrium involved the second-mover dropping out of the contest. However, we did not wish $a$ to be set at so high a value that it would make the outcome of the game trivial.

Table 1 outlines the different treatments as well as the predictions for each one. The

\footnotetext{
${ }^{9}$ In the experiment, this is equivalent to Player 1's bid variable being given a weight of 7 and Player 2's bid variable having a weight of 3 .
} 
Table 1: Theoretical predictions.

\begin{tabular}{cccccc}
\hline $\begin{array}{c}\text { Treatment } \\
\text { Name }\end{array}$ & Timing & Symmetry & $\begin{array}{c}\text { Bids } \\
\text { (Player 1, Player 2) }\end{array}$ & $\begin{array}{c}\text { E(Payoff) } \\
\text { (Player 1, Player 2) }\end{array}$ & $\begin{array}{c}\text { Rent } \\
\text { Dissipation }\end{array}$ \\
\hline \hline simsym & Simultaneous & Symmetric & $(50,50)$ & $(50,50)$ & 100 \\
simasym & Simultaneous & Asymmetric & $(42,42)$ & $(98,18)$ & 84 \\
seqsym & Sequential & Symmetric & $(50,50)$ & $(50,50)$ & 100 \\
seqasym & Sequential & Asymmetric & $(86,0)$ & $(114,0)$ & 86 \\
\hline
\end{tabular}

introduction of asymmetry in the simultaneous-move game results in a relatively small change in equilibrium effort level (drop by 8 units or 16\% for both players) but that has a very large impact in terms of expected payoff (goes up by 48 units (96\%) for the first-mover and decreases by 32 units (64\%) for the second-mover.) This is despite the fact that both players submit the same effort in equilibrium.

The same asymmetry has an even more dramatic effect in the sequential-move game. Here, both the equilibrium effort and expected payoff to the first-mover rise - effort goes up by 36 units (72\% and expected payoff rises by a massive 64 units (128\%). In contrast, both the equilibrium effort level and the expected payoff of the second-mover fall to zero. Based on the risk-neutral Nash equilibrium predictions, we are still able to establish a number of testable hypotheses.

Hypothesis 1: In simultaneous-move treatments, average bids by symmetric bidders will be higher than either type of asymmetric bidders.

Hypothesis 2: Average bids across types will be equal in the simasym treatment.

Hypothesis 3: If given a first-mover advantage, more able players will submit a higher level of effort than in the simultaneous-move case.

Hypothesis 4: In the seqasym treatment, the first-mover will bid an amount which will deter the second-mover from entering the contest.

Hypothesis 5: In both timing protocols, more able players will earn higher expected profits than both symmetric and less able players.

Hypothesis 6: First-mover advantage only exists (in expected payoff terms) when there is also a ability advantage.

The experimental design consisted of the following. The experiment was programmed and conducted with the software z-Tree (Fischbacher, 2007). Three sessions were run for each treatment. 
Ten subjects took part in each experimental session. Each individual took part in only one session and no subject had participated in similar experiments.

Upon arrival at the lab, subjects were invited to take a seat in a booth which did not allow for any visual communication between subjects. Once all subjects were seated, they received a written copy of the instruction set; at this point the experimenter requested that no verbal communication take place. The experimenter read the instruction set aloud to ensure common knowledge of the experimental conditions. The five subsequent minutes were allocated for a detailed study of the instruction sets and possible questions. The experiment commenced once all questions raised by subjects were answered in private.

In both symmetric and asymmetric experiments, each subject was told he was taking part in an experiment that would last 30 periods. Subjects were assigned to one of two types of persons, A and B. Subjects retained their roles throughout the experiment. In each period, all subjects received 300 Experimental Currency Units (ECU) and a type-A person was randomly matched with a type-B person. Subjects could use any part of their endowment to bid for a prize valued at 200 ECU. ${ }^{10}$ The more they bid, the more likely they were to win the prize; the more the other person bid, the less likely they were to win. More specifically, in the symmetric treatments, subjects were told that each ECU spent by an A person would be converted into a blue ball and each ECU spent by a B person would be converted into a red ball. All the balls would be put in a bag and the computer would draw a ball at random. If a blue ball was drawn, subject A would win; if a red ball was drawn, subject B would win. In the asymmetric treatments, each ECU spent by the type-A person would count as 7 blue balls, while each ECU spent by a type-B person would count as 3 red balls. They were also informed that they would forfeit their bids regardless of winning or not.

The decision screen provided information about the value of the prize and their endowment in every period. It also had a field where subjects typed how much of their endowment they wanted to invest in that period. In order to assist subjects with computing probabilities of victory, part of the screen displayed a calculator which computed the winning probability for the subject given any pair of hypothetical effort levels for both players. Subjects were free to calculate different combinations of bids as many times as they wished in each period. The calculator was also available in every period. ${ }^{11}$

\footnotetext{
${ }^{10}$ Subjects's endowments were equal to 300 ECU in order to prevent negative payoffs.

${ }^{11}$ The use of the calculator was indeed frequent in the early stages of each session by all subjects. However, its
} 
Table 2: Average effort levels over time - simsym and simasym treatments

\begin{tabular}{ccccc}
\hline Periods & $1-10$ & $11-20$ & $21-30$ & All \\
\hline \hline E (Symmetric bidders) & 119.50 & 95.36 & 85.40 & 100.08 \\
& $(75.96)$ & $(63.45)$ & $(52.54)$ & $(66.19)$ \\
$\mathrm{N}$ & 300 & 300 & 300 & 900 \\
E ("Weak" bidders) & 100.93 & 69.12 & 61.35 & 77.13 \\
$\mathrm{~N}$ & $(95.42)$ & $(76.70)$ & $(57.00)$ & $(79.66)$ \\
E ("Strong" bidders) & 150 & 150 & 150 & 450 \\
& $(85.56)$ & $(69.68)$ & $(39.87)$ & $(71.12)$ \\
$\mathrm{N}$ & 150 & 150 & 150 & 450 \\
\hline
\end{tabular}

Standard deviations in parenthesis.

At the end of each period, a screen informed subjects of their bid, the bid of the subject with whom they were paired, whether they had won or not and their payoff for the period. Payments consisted of the accumulated earnings throughout the experiment. One thousand ECU was worth $£ 1$ (\$1.74). Each session lasted about an hour and the subjects' average payment was $£ 11.40$ (\$19.84). All sessions were conducted between September and November 2003 in the Experimental Economics Laboratory at Royal Holloway University of London.

\section{Experimental Results}

\subsection{Simultaneous-move Games}

We begin by analyzing the simultaneous-move treatments. For the asymmetric treatments, we shall henceforth denote type-A players as "strong" players and the type-B players as "weak." Table 2 gives average bids for subjects in both the symmetric and asymmetric treatments. There is a steady decline in average bids over the course of the experiments. It is also noticeable that the average bid for the symmetrical players is always above the average bids for the two types of asymmetric players. Average bids in the initial stages of the experiments were much higher than the predictions for both treatments. However, a sharp decline not only in average bids but also in their standard deviations is also noticeable for all types of subjects over the course of the sessions. The disperse nature of the data for the initial periods can be attributed to subjects starting to become acquainted with the experimental protocol (i.e. the random matching procedure,) as well as learning what the frequency of use declined as the experiments progressed. 
optimal strategy is. ${ }^{12}$ Taking a conservative approach, it is fair to assume that by the twentieth period most subjects will have fully understood the game. Therefore in the data analysis, unless noted, all the data analysis refers to the last 10 periods of the experiment. ${ }^{13}$

Closer observation of table 2 shows that average bids on the last third of the experiments are still higher than predictions for both treatments. In addition, average bids for both types of bidders in the asymmetric case are remarkably close (61.35 for the "weak" bidders and 62.08 for the "strong" bidders) and they are not statistically different(F-test, $\mathrm{p}=0.897)$. Average bids in the simsym treatment are also higher than predicted; when compared with the asymmetric bidders, the average bids in simsym appear to be much higher than the average bids in the simasym (t-test, $p<0.001)$. These are our first two results.

Finding 1: Average effort levels in the simultaneous-move treatments are above predictions in both symmetric and asymmetric cases.

Finding 2: Average effort level by subjects in the symmetric treatment is higher than either type of subject in the asymmetric treatment.

In order to better understand the subjects' behavior, we now look at the distribution of bids in the last ten periods. Table 3 displays a histogram of the effort levels in both simsym and simasym treatments. The modal effort interval in the simsym treatment is $[61,80]$ with $23 \%$ of observations. The interval which includes the RNNE has $17 \%$ of all observations. It is worth noticing that $66 \%$ of all observations record effort levels larger than 60 ECU. In the seqasym treatment, the modal effort interval is [41-60], which includes the RNNE prediction. However, we see a large proportion of observations recording effort levels either below (40\%) or above (42\%) the equilibrium. Breaking up the data by player type, we see that almost a third of the "weak" players is bidding no more

\footnotetext{
${ }^{12}$ We note that the stochastic nature of the Tullock function makes learning somewhat more difficult vis-à-vis other type of contest success functions. This is because submitting a higher effort level than your opponent does not mean you will win for certain, as opposed to a perfectly discriminating contest, where the highest bidder wins.

${ }^{13}$ In order to conduct significance tests for the mean values, we run OLS regressions of the form $E=\beta_{0}+\beta_{1} D_{i}+\epsilon_{i}$. The dependent variable, $E$ is individual subjects' average effort level across the last ten periods and $D_{i}$ is a dummy variable accounting for the type of bidder (e.g. "strong" or "weak.") The estimate of $\beta_{1}$ can therefore be interpreted as the difference in means. Significance tests will consist of standard t- and F-tests. To avoid the problem of nonindependence of observations within sessions, we use White-adjusted standard errors (White, 1980). A complete breakdown of the regression output is available upon request. For presentational reasons, we will refer only to the significance levels of the appropriate test.
} 
Table 3: Relative frequency of effort levels in simsym and simasym treatments

\begin{tabular}{ccccccc}
\hline \hline $\begin{array}{c}\text { Effort } \\
\text { Range }\end{array}$ & $\begin{array}{c}\text { Symmetric } \\
\text { Bidders }\end{array}$ & $\begin{array}{c}\text { F\&S } \\
\text { Prediction }\end{array}$ & $\begin{array}{c}\text { Asymmetric } \\
\text { Bidders }\end{array}$ & $\begin{array}{c}\text { F\&S } \\
\text { Prediction }\end{array}$ & $\begin{array}{c}\text { Strong } \\
\text { Bidders }\end{array}$ & $\begin{array}{c}\text { Weak } \\
\text { Bidders }\end{array}$ \\
\hline $0-20$ & 0.09 & 0.00 & 0.26 & 0.00 & 0.20 & 0.32 \\
$21-40$ & 0.08 & 0.16 & 0.14 & 0.27 & 0.17 & 0.12 \\
$41-60$ & 0.17 & 0.33 & 0.18 & 0.41 & 0.13 & 0.22 \\
$61-80$ & 0.23 & 0.29 & 0.09 & 0.27 & 0.14 & 0.05 \\
$81-100$ & 0.17 & 0.22 & 0.13 & 0.05 & 0.20 & 0.05 \\
$101-120$ & 0.06 & 0.00 & 0.10 & 0.00 & 0.13 & 0.07 \\
$121-140$ & 0.04 & 0.00 & 0.02 & 0.00 & 0.02 & 0.02 \\
$141-160$ & 0.04 & 0.00 & 0.05 & 0.00 & 0.01 & 0.09 \\
$161-180$ & 0.03 & 0.00 & 0.01 & 0.00 & 0.00 & 0.02 \\
$181-200$ & 0.06 & 0.00 & 0.02 & 0.00 & 0.01 & 0.03 \\
$201-300$ & 0.01 & 0.00 & 0.01 & 0.00 & 0.00 & 0.01 \\
\hline \hline
\end{tabular}

than 20 ECU, which is the modal effort interval. The distribution of effort by "strong" bidders has two modes, at the $[0,20]$ and $[81,100]$ range of bids. Effort levels up to 100 account for $83 \%$ of all bidding by "strong" bidders.

The table also includes the predicted effort levels if we assume that players are inequality averse with Fehr and Schmidt (1999) preferences (F\&S). ${ }^{14}$ Based on the estimates of Blanco et al. (2007), we constructed a distribution of predicted effort levels for seqsym and seqasym. On one hand, levels of effort above the RNNE are rationalized by the existence of more competitive players (with higher $\alpha$ ). If one recalls equation $9, \delta E_{i} / \delta \alpha>0$, which means that the more competitive a player is, the more effort he will submit in the equilibrium of the simultaneous-move game. On the other hand, the observations recording effort levels below the RNNE can be explained by a proportion of subjects who are averse to advantageous inequality $(\beta>0)$, since $\delta E_{i} / \delta \beta<0$, which implies that the more cooperative a player is, the less effort he will put in equilibrium. Nevertheless, the theory still fails to predict effort levels above 100 ECU, even if we were to assume extreme levels of $\alpha$.

\footnotetext{
${ }^{14}$ Fehr and Schmidt (1999) propose that in any given population there are various types of players. The distribution of types of players is a function of a joint distribution of $\alpha$ and $\beta$. Blanco et al. (2007) estimate the two parameters based on a variety of games. In their sample, $31 \%$ of subjects have $0 \leq \alpha<0.4,33 \%$ with $0.4 \leq \alpha<0.92,23 \%$ with $0.92 \leq \alpha<4.5$ and $13 \%$ with $4.5 \leq \alpha$. Also, they find that $29 \%$ of their sample has $0 \leq \beta<0.235,15 \%$ of the sample with $0.235 \leq \beta<0.5$ and $56 \%$ with $0.5 \leq \beta$. If we apply these estimates to equation 9 , recalling that $\beta \leq \alpha$, we obtain the distributions outlined in table 3.
} 
Table 4: Average effort levels by first-movers in seqsym and seqasym treatments

\begin{tabular}{ccccc}
\hline Periods & $1-10$ & $11-20$ & $21-30$ & All \\
\hline \hline seqsym & 79.77 & 88.44 & 80.29 & 82.83 \\
& $(72.63)$ & $(54.60)$ & $(54.73)$ & $(61.24)$ \\
$\mathrm{N}$ & 150 & 150 & 150 & 450 \\
seqasym & 99.01 & 71.45 & 64.13 & 78.20 \\
& $(71.12)$ & $(42.85)$ & $(30.03)$ & $(53.04)$ \\
$\mathrm{N}$ & 150 & 150 & 150 & 450 \\
\hline \multicolumn{5}{r}{ Standard deviations in parenthesis }
\end{tabular}

\subsection{Sequential-move Games}

Table 4 depicts the average effort levels and their standard deviations by first-movers in both sequential-move treatments throughout the course of the experiments. While in seqsym, average effort oscillates between 80 and 90 units, the data from the seqasym treatment shows a substantial drop in average effort level from just below 100 units to 64 . This indicates that in the symmetric treatment, average first-mover effort level is above prediction (F-test, $p<0.001$ ), while in the asymmetric treatment, it is below prediction (F-test, $p<0.001$ ). Both treatments show a drop in the standard deviation, which indicates that behavior is becoming less noisy as the experiments progress, although the degree of dispersion is still quite high for the symmetric treatment. Asymmetric first-movers submit a higher level of effort than more able players in the simultaneous-move case, although the difference is not significant.

Finding 3: Asymmetric first-movers do not submit statistically higher levels of effort than strong players in the simultaneous-move treatment.

The modal choice range by first-movers in seqsym is between 81 and 100, well above the prediction of 50. However the second most chosen effort range is [41,60]. Also, it is worth noting that only just over a quarter $(26.67 \%)$ of observations show first-movers choosing effort levels above 100. In seqasym, the majority of bids (78.73\%) are between 41 and 100 and its distribution has two modes, one is the interval of bids between 81 and 100, which includes the Nash equilibrium bid of this game (86). The other encompasses bids between 41 and 60 .

Since second-movers were informed of the actions of the first-movers, it makes more sense to analyze their behavior conditional on the effort level chosen by the first-movers. Figure shows second movers' bids as a function of first-movers effort levels in both sequential-move treatments. The $\mathrm{x}$-axis measures first-mover bids and the $\mathrm{y}$-axis measures second-mover bids. The figure displays both observed effort level pairs for the last ten periods, the predicted best-reply function and the 
estimated best-reply function from the data.

In the seqsym treatment, judging from the estimated best-reply function, it would appear that second-movers seem to on average overbid relative to the risk-neutral best-reply. However, the scatter plot reveals that second-movers either overbid first-movers or drop out of the contest altogether. Additionally, their bidding levels are much higher than would be predicted by a riskneutral best-reply function for any range of first-mover bids, which is quite clear when we observe the estimated best-reply function. The average absolute per period difference from observed bid and the risk-neutral best-reply is 53.88, which implies a loss of 26.27 ECU in expected payoff.

In the seqasym treatment, we see a very large cluster of observations (66 observations, 39\% of all observations in the last 10 periods) where the first-movers bid a level of effort between 60 and 100 to which the second-movers respond by bidding a value close to or equal to zero. However, there are a large number of observations where second-movers bid very large amounts in response to first-movers. This leads to the estimated best-reply function to always be above the theoretical best-reply function. Second-movers are again not maximizing their expected value of the contest. The average difference per period between the risk-neutral best-reply and the observed reply was 45.10. This had an associated loss in expected value terms of 17.15. The introduction of the asymmetry helps subjects to coordinate on the SPNE, since aggressive replies by second-movers become more "expensive".

Inequality aversion can rationalize part of the range of responses by second-movers in both treatments. With these preferences, the best-reply function for Player 2 would be: $E_{2}=-a E_{1}+$ $\sqrt{\frac{\left(a E_{1} J\right)(1+2 \alpha-2 \beta)}{1+\alpha-\beta}}$. Since $\frac{\delta E_{2}}{\delta \alpha}>0$, the more averse Player 2 is to disadvantageous inequality, the more effort he will put in response to Player 1. Conversely, the higher the degree of advantageous inequality aversion, the lower Player 2's response will be to Player 1's action, since $\frac{\delta E_{2}}{\delta \beta}<0$. It is straightforward to show that the minimum level of effort based on any combination of $\alpha$ and $\beta$ by second-movers is the standard best-reply function. The minimum level $\alpha$ can take is zero and the model assumes $\beta \leq \alpha$. Conversely, the maximum best-reply to any effort level by firstmovers is found by setting $\beta=0$ and $\alpha=4.5$. The grey line in both figures shows the highest possible best-reply function under Fehr and Schmidt preferences. In seqsym, the estimated bestreply function is very close to the maximum best-reply predicted by inequality aversion. In seqasym, the estimated best-reply is between the standard prediction and the maximum best-reply under inequality aversion. The introduction of ability asymmetry leads to average behavior being less competitive. This could be due to the fact that the asymmetry in ability makes punishing high 

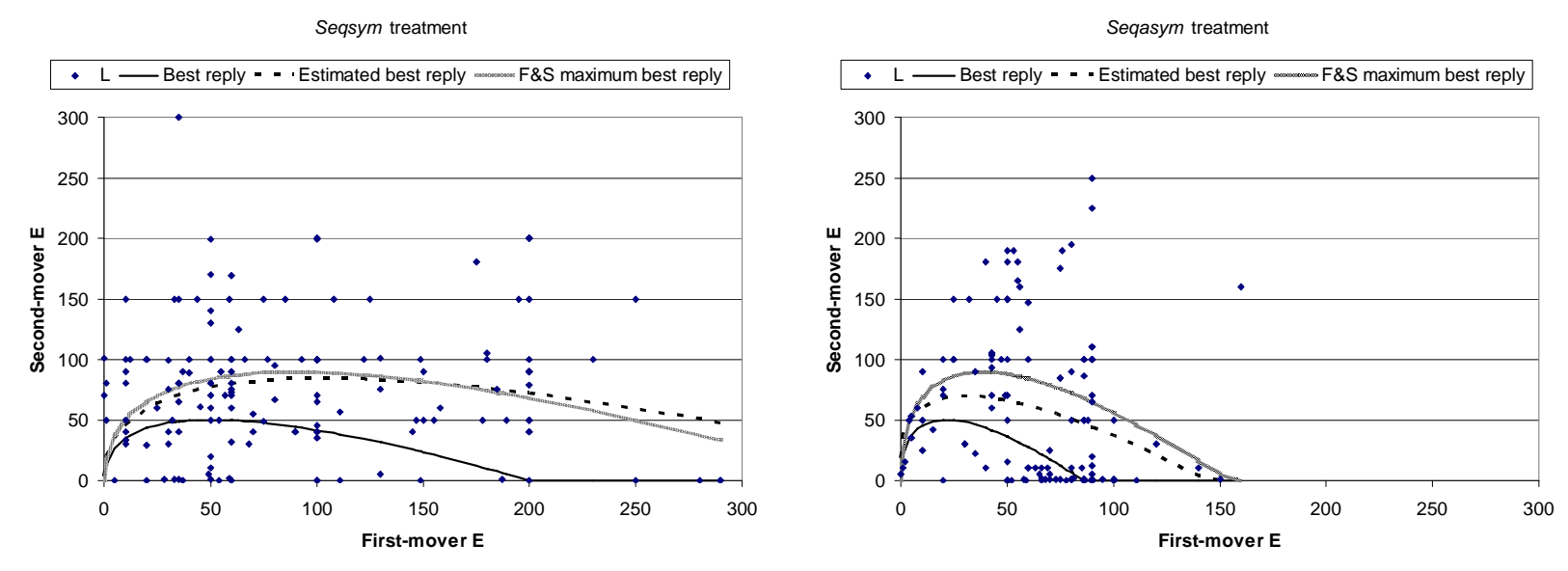

Figure 2: Plot of the distribution of followers' bids conditional on leaders' bids - seqasym and seqasym treatments

first-mover effort by overbidding very costly. Nevertheless, it should be noted that even extreme competitive behavior as expressed by a high $\alpha$ parameter cannot explain the very high levels of bidding by second-movers.

Finding 4: A large proportion of first-movers with an ability advantage is able to deter the less able second-movers from entering the contest. However, a substantial proportion of secondmovers responds by submitting very large bids.

Finally we address whether there is a first-mover advantage based on the choices made by subjects. Given that subjects' effort decisions are intended to increase the likelihood of winning a prize, their choices ought to be guided by expected profits, rather than realized profits. Hence, it is only appropriate to analyze expected profits, rather than realized profits. The latter would be useful were we to analyze learning over the course of the experiment. We calculated the expected profit for each subject for each of the last ten periods based on the choices made by him and the other player and regressed that against a set of dummy variables indicating the type of subject. The variable codes indicate the treatment type. The first three letters indicate the timing protocol, SIM if simultaneous-move treatment and SEQ if sequential-move treatment. In the asymmetric cases, STR indicates a "strong" player, while WK indicates a "weak" player. Finally, SEQSYML and SEQSYMF indicate first- and second-movers, respectively in the seqsym treatment. The interpretation of the dummy coefficient is therefore the difference in means vis-à-vis the omitted 
Table 5: OLS results for treatment differences in expected payoffs

\begin{tabular}{|c|c|}
\hline Dependent variable & $\overline{\mathrm{E} \text { (Payoff) }}$ \\
\hline \multirow[t]{2}{*}{ Constant } & $14.60^{\dagger}$ \\
\hline & $(2.56)$ \\
\hline \multirow[t]{2}{*}{ SIMSTR } & $59.39^{\dagger}$ \\
\hline & $(4.41)$ \\
\hline \multirow{2}{*}{ SIMWK } & $-12.01^{\dagger}$ \\
\hline & $(4.52)$ \\
\hline \multirow[t]{2}{*}{ SEQSYML } & 0.96 \\
\hline & $(4.26)$ \\
\hline \multirow[t]{2}{*}{ SEQSYMF } & 0.80 \\
\hline & $(4.56)$ \\
\hline \multirow[t]{2}{*}{ SEQSTR } & $68.25^{\dagger}$ \\
\hline & $(4.25)$ \\
\hline \multirow[t]{2}{*}{ SEQWK } & $-15.04^{\dagger}$ \\
\hline & $(4.41)$ \\
\hline $\mathrm{N}=1200$ & R-squared $=0.32$ \\
\hline
\end{tabular}

category, the simsym treatment. As expected due to the large overbidding, the constant value, which determines the expected payoff in the simsym treatment is significantly below the prediction of 50 (F-test, $p<0.00001$.) The data seems to support the theoretical prediction that there is neither advantage in moving first nor a disadvantage in moving last compared to a simultaneous move game if players are symmetric, as the coefficients on SEQSYML and SEQSYMF are close to zero and insignificant.

By contrast, in the asymmetric treatments being the "strong" player yields a substantial advantage in expected payoff terms, even more so if the "strong" player also moves first (F test, $p=0.073)$. Conversely, being the "weak" player results in a loss in expected payoff, although being a second-mover does not result in further loss of expected payoff vis-à-vis the simsym treatment (F-test, $p=0.559$ ). Also note that the values of the coefficients reflect the expected payoff losses resulting from the deviations from the risk-neutral predictions. In short, despite the fact that subjects do not bid as expected, the theory predicts well the effect of a change from a symmetric game to an asymmetric one in terms of expected profits. We conclude this section with the following findings.

Finding 5: The introduction of ability (dis)advantage leads to (lower)higher expected profits vis-à-vis the symmetric case. 
Finding 6: First-mover advantage is only observed in the presence of asymmetry in the contest success function.

\section{Discussion}

This study seeks to understand the impact of asymmetries on behavior in Tullock contests. We consider two different protocols: one where both players move simultaneously and another where the timing of moves is sequential. The predicted impact of introducing asymmetries in the Tullock success function was markedly different for the two timing protocols. In the simultaneous-move case, the aggregate level of effort would decline, driven by an equal fall in effort levels by both players. In the sequential-move case, the aggregate effort level should also fall, but here solely at the expense of the "weak" player, who is expected to drop out of the contest in reply to a large effort outlay by the "strong" first-mover. We observe a substantial level of overbidding relative to risk-neutral Nash predictions. This is in line with previous studies of Tullock contests and all-pay auctions (Millner and Pratt, 1991; Davis and Reilly, 1998), as well as other types of contests like tournaments (Müller and Schotter, 2003).

Nevertheless, the theory seems to predict well the qualitative impact of changes in the contest function on expected payoffs for both timing protocols, even if the data does not corroborate the point predictions regarding bid levels. More apt subjects are able to extract higher expected rents than their symmetric counterparts. Furthermore, if given the opportunity to move first, more able players succeed in a large number of cases in excluding the second-mover from the contest and extract more expected profits than in the simultaneous-move case. However, more able firstmovers do not exert more effort than their counterparts in the simultaneous-move case, contrary to theoretical predictions (Dixit, 1987).

This nevertheless indicates that there is an advantage to being an incumbent in terms of expected profitability if one is sufficiently more able than the competition. This is particularly relevant for $\mathrm{R} \& \mathrm{D}$ competition; the type of contest considered in this paper would correspond to an $R \& D$ race where there are no technological spill-overs between competitors. The prize is the discounted value of the profit streams afforded by the patent. The probability of achieving the patent first is solely determined by how much each firm invests in product development and the relative know-how of each research department. An incumbent advantage can exist if the firm has previous experience in research in the pertinent area vis-à-vis a new entrant. Our evidence suggests 
that if the incumbent advantage is coupled with significant expertise, it may prevent the $R \& D$ race from ever taking place.

This evidence is consistent with Dasgupta and Stiglitz (1980)'s analysis. The authors defend that it is always in the interest of a monopolist to preempt $\mathrm{R} \& \mathrm{D}$ competition. If another firm is able to obtain a patent, the industry would become a duopoly with the corresponding decrease in profitability. Hence, the first-mover advantage allows the incumbent monopolist to prevent entry by committing to R\&D expenditures at a level which makes entry unprofitable for the other firm. Our results show that this is accomplished without significant increases in expenditures.

In a different context, if one views rent-seeking in terms of efforts to maintain a monopoly position, such as an external consulting contract, first-mover advantage may be beneficial in the sense that it prevents other firms from engaging in wasteful expenditures. However, the results do not offer a clear cut case for the use of neither the simultaneous- nor sequential-move protocols, since aggregate rent dissipation is roughly the same for both.

It is pertinent to contrast our evidence with that of the only other study of sequential contests of which we are aware (Weimann et al., 2000), in which each player's effort level is raised to the power of 8 . Theory also predicts the first-mover to commit to a level of effort such that the second-mover does not find it profitable to enter. The authors find no evidence of first-movers being able to commit to a level of effort which deters second-movers from entering the contest. Whenever first-movers commit to high levels of effort, second-movers punish them by exerting equally high levels of effort, such that first-movers' (expected) payoffs equal zero. It certainly is the case that some second-movers in our study also behave in a similar fashion. Nevertheless, we also find a large proportion of observations where "strong" first-movers successfully deter "weak" second-movers. In this sense, one can argue that ability asymmetry is a more powerful commitment device than high effectiveness of effort.

There are a number of possible explanations for this phenomenon. By allowing players to have a disutility from expected payoff differentials, we are able to rationalize to some extent why there would be subjects who bid below or above the risk-neutral Nash equilibrium level. The higher the disutility from disadvantageous inequality, the more effort a subject will put into the contest; in other words, the more competitive that subject will be. One can also interpret the excess bidding as a punitive device in the sequential-move game. Such behavior has been reported in other experimental studies of sequential-move games, such as quantity-setting oligopolies (Huck, Müller and Normann, 2002), where the excess bidding by second-movers will explicitly reduce 
the expected payoff of the first-mover. Conversely, the higher the disutility from advantageous inequality, the less effort a subject will put into the contest. This would imply that the subject is more cooperative. This line of reasoning has intuitive appeal and is able to explain a substantial proportion of the data ( $80 \%$ of all observations). However, it fails to rationalize the extreme overbidding observed in roughly $20 \%$ of the data across treatments. If behavior in the experiment is indeed driven by inequality aversion, our evidence is in contrast with Konrad (2004), in that subjects earn significantly less than a purely self-interested subject would earn.

Risk aversion, although prominently used to explain behavior in other settings like auctions, has little predictive power in Tullock contests without making very stringent assumptions on behavior. Skaperdas and Gan (1995) show that if subjects are either risk-neutral or have utility functions which exhibit Constant Absolute Risk Aversion, equilibria exist where the less risk-averse player should spend more effort in equilibrium than the more risk-averse player. The authors argue that the less risk-averse player will insure himself against the losing the contest by outbidding his opponent. This theoretical insight is corroborated by the only study of the effects of risk aversion on behavior in Tullock contests (Millner and Pratt, 1991). However, it is not possible to measure the extent to which this excess bidding is due to risk aversion, as appropriate measures of risk preferences are unavailable in the current dataset.

A final rationale for the excess bidding we observe in the data comes from the "joy-ofwinning' hypothesis. The introduction of an exogenous utility from winning the contest causes the best-reply function to fan out; this in turn leads to a higher effort level in equilibrium. This hypothesis has been successful in explaining behavior in first-price auctions (Cox, Smith and Walker, 1988).

The dichotomy in effort levels between those who choose to submit either very low or very high levels of effort has also been reported in tournament settings. Müller and Schotter (2003) test in the lab a variant of an n-player, multi-unit all-pay first-price auction, where effort has an addition noise component. In this game, risk aversion results in a lower level of effort relative to the risk-neutral case. The authors find that whenever there is heterogeneous ability, the high ability players submitted high levels of effort, whilst the low ability players dropped out. Unlike Müller and Schotter (2003), we find that dichotomy in effort is present not only across ability levels but also within players of similar ability. Our evidence suggests that individual ability is not an impediment for an employee to try hard and succeed in the contest. However, considering high-ability employees first may deter some low-ability employees from taking part. 


\section{References}

[1] Blanco, M. Engelmann, D., and Normann, H-T., 2007. A within-subject analysis of otherregarding preferences. Mimeo.

[2] Bull, C., Schotter, A. and Weigelt, K., 1987. Tournaments and piece rates: an experimental study. Journal of Political Economy 95(1), 1-33.

[3] Coughlan, P.J. and Plott, C.R., 2003. An experimental analysis of the structure of legal fees: American rule vs. English rule. Social Science Working Paper no. 1025. Pasadena: California Institute of Technology.

[4] Cox, J.C., Smith, V.L. and Walker, J.M., 1988. Theory and individual behavior of first-price auctions. Journal of Risk and Uncertainty 1. 61-99.

[5] Dasgupta, P., and Stiglitz, J. 1980. Uncertainty, industrial structure and the speed of R\&D. The Bell Journal of Economics 11, 1-28.

[6] Davis, D.D. and Reilly, R.J., 1998. Do too many cooks always spoil the stew? An experimental analysis of rent-seeking and the role of a strategic buyer. Public Choice 95, 89-115.

[7] Dixit, A., 1987. Strategic behavior in contests. American Economic Review 77, 891-898.

[8] Fehr, E., and Schmidt, K. 1999. A theory of fairness, competition and cooperation. Quarterly Journal of Economics 114, 817-868.

[9] Fischbacher, U., 2007. Z-Tree: Zurich Toolbox for Readymade Economic Experiments, Experimental Economics 10, 171-178.

[10] Hirshleifer, J. and Osbourne, E., 2001. Truth, effort and the legal battle. Public Choice 108, 169-195.

[11] Huck, S., Müller, W. and Normann, H-T., 2002. To commit or not to commit: endogenous timing in experimental duopoly markets. Games and Economic Behavior 38, 240-264.

[12] Kagel, J.H., 1995. Auctions: A Survey of Experimental Research, in The Handbook of Experimental Economics, edited by J.H. Kagel and A.E. Roth, Princeton University Press.

[13] Konrad, K.A., 2004. Altruism and envy in contests: an evolutionary stable symbiosis. Social Choice and Welfare 22, 479-490. 
[14] Konrad, K.A. and Schlesinger, H., 1997. Risk aversion in rent-seeking and rent-augmenting games. Economic Journal 107, 1671-1683.

[15] Leininger, W. 1993. More efficient rent-seeking - a Münchhausen solution. Public Choice 75, 43-62.

[16] Lockhard, A.A. and Tullock, G., 2001. Efficient rent seeking: chronicle of an intellectual quagmire, Kluwer Academic Publisher.

[17] Loury, G., 1979. Market structure and innovation. Quarterly Journal of Economics 93, 395410.

[18] Millner, E.L. and Pratt, M.D., 1989. An experimental investigation of efficient rent-seeking. Public Choice 62, 139-151.

[19] Millner, E.L. and Pratt, M.D., 1991. Risk aversion and rent-seeking: An extension and some experimental evidence. Public Choice 69, 81-92.

[20] Müller, W. and Schotter, A., 2003. Workaholics and drop outs in optimal organizations. mimeo.

[21] O'Keefe, M., Viscusi, W.K., and Zeckhauser, R.J., 1984. Economic Contests: comparative reward schemes. Journal of Labor Economics 2, 27-56.

[22] Schotter, A. and Weigelt, K., 1992. Asymmetric Tournaments, Equal Opportunity Laws and Affirmative Action: Some Experimental Results. Quarterly Journal of Economics 107, 511-539.

[23] Skaperdas, S. and Gan, L., 1995. Risk aversion in contests. Economic Journal 105, 951-962.

[24] Tullock, G., 1980. Rent-seeking as a zero-sum game. In: J.M. Buchanan, R.D. Tollison and G. Tullock (Eds), Toward a Theory of the rent-seeking society. College Station: Texas A\&M University Press.

[25] Weimann, J., Yang, C. and Vogt, C., 2000. An experiment on sequential rent seeking. Journal of Economic Behavior and Organization 41, 405-426.

[26] White, H., 1980. A heteroskedasticity-consistent covariance matrix estimator and a direct test for heteroskedasticity. Econometrica 48, 817-930. 


\section{A Sample Instruction Sets}

\section{Simultaneous Asymmetric Treatment Instructions}

Welcome to our experiment! Please read these instructions very carefully! Do not talk to your neighbours and keep quiet during the entire experiment. If you have any questions, please raise your hand, and we will answer your question privately.

In this experiment you will be given an endowment of 300 Experimental Currency Units (ECU) in every period. You will use any part of your endowment to bid for a prize. There will be another person bidding for the same prize. This prize is worth $200 \mathrm{ECU}$ to you and the other person. Importantly, regardless of who wins, both persons will have to pay their bids.

So if you win the prize, your payoff is equal to your endowment plus the prize minus your bid. If you lose, your payoff will be equal to your endowment minus your bid. Note that in the event that both persons bid zero, neither of them will win the prize.

If you win: Payoff $=$ Endowment + Prize - Bid

If you lose: Payoff $=$ Endowment - Bid

The experiment will work as follows: there will be two types of persons, type A and type B persons. There are as many type-A persons as there are type-B persons in the room. To find out what type of person you are, please check the top right-hand corner of this instruction set.

In every round, a type-A person is randomly matched with a type-B person, and both persons will decide how many ECU's to bid.

The more you bid, the more likely you are to win. The more the other person bids the less likely you are to win. Specifically, each ECU a type-A person bids counts as 3 blue balls and each ECU a type-B person bids counts as 7 red balls. All red and blue balls are put into an opaque bag, and one is taken at random. If the chosen ball is blue, the type A person wins the prize, if the chosen ball is red, the type B person wins the prize.

To help you compute how likely you are to win the prize, we provide you with a probability calculator. It tells you the probability of winning the prize given the bids made by you and the other person. You can compute this as many times as you wish.

After both persons have made their decisions, the winner will be decided and you will receive information about your payoffs on a separate screen.

There will be 30 periods in this experiment. At the end of the experiment, we will calculate the sum of your earnings and pay them to you in cash. 1000 ECU are worth $£ 1.20$. 


\section{Sequential Symmetric Treatment Instructions}

Welcome to our experiment! Please read these instructions very carefully! Do not talk to your neighbours and keep quiet during the entire experiment. If you have any questions, please raise your hand, and we will answer your question privately.

In this experiment you will be given an endowment of 300 Experimental Currency Units (ECU) in every period. You will use any part of your endowment to bid for a prize. There will be another person bidding for the same prize. This prize is worth $200 \mathrm{ECU}$ to you and the other person. Importantly, regardless of who wins, both persons will have to pay their bids.

So if you win the prize, your payoff is equal to your endowment plus the prize minus your bid. If you lose, your payoff will be equal to your endowment minus your bid. Note that in the event that both persons bid zero, neither of them will win the prize.

If you win: Payoff $=$ Endowment + Prize - Bid

If you lose: Payoff $=$ Endowment - Bid

The experiment will work as follows: there will be two types of persons, type A and type B persons. There are as many type-A persons as there are type-B persons in the room. To find out what type of person you are, please check the top right-hand corner of this instruction set.

In every round, a type-A person is randomly matched with a type-B person. Then in the first part of the round, the type-A persons will decide how much they should bid. After the type-A person have submitted their bid, it will be the turn of the type-B persons to make their decision after knowing the bid of the type-A their are matched with.

The more you bid, the more likely you are to win. The more the other person bids the less likely you are to win. Specifically, each ECU a type-A person bids counts as 1 blue ball and each ECU a type-B person bids counts as 1 red ball. All red and blue balls are put into an opaque bag, and one is taken at random. If the chosen ball is blue, the type A person wins the prize, if the chosen ball is red, the type B person wins the prize.

To help you compute how likely you are to win the prize, we provide you with a probability calculator. It tells you the probability of winning the prize given the bids made by you and the other person. You can compute this as many times as you wish.

After type-B persons have made their decisions, the winner will be decided and you will receive information about your payoffs on a separate screen.

There will be 30 periods in this experiment. At the end of the experiment, we will calculate the sum of your earnings and pay them to you in cash. 1000 ECU are worth $£ 1.20$. 
Table 6: OLS output on difference in effort levels between simsym and simasym subjects.

\begin{tabular}{cc}
\hline \hline Dependent variable & $\mathrm{E}$ \\
\hline Constant & $85.40^{\dagger}$ \\
& $(3.04)$ \\
STR & $-23.32^{\dagger}$ \\
& $(4.45)$ \\
$\mathrm{WK}$ & $-24.05^{\dagger}$ \\
& $(5.55)$ \\
$\mathrm{N}=600$ & $\mathrm{R}$-squared $=0.051$ \\
\hline \hline Robust standard errors in parenthesis. \\
${ }^{\dagger}$ indicates coefficient is statistically significant at the $1 \%$ level.
\end{tabular}

Table 7: OLS output on difference in effort levels between seqsym and seqasym first-movers.

\begin{tabular}{cc}
\hline \hline Dependent variable & $\mathrm{E}$ \\
\hline Constant & $80.29^{\dagger}$ \\
& $(2.98)$ \\
ASYM & $-16.16^{*}$ \\
& $(7.32)$ \\
$\mathrm{N}=300$ & R-squared $=0.032$ \\
\hline \hline Robust standard errors in parenthesis. & \\
$\dagger$ and ${ }^{*}$ indicate coefficient is statistically significant at the $1 \%$ and $10 \%$ level, respectively.
\end{tabular}

\section{B Regression output - not for publication.}

